\title{
Brain and Body: Mitochondrial Disease, Fever, and Impulsivity
}

\author{
By Eric Hollander, MD
}

As spring turns to summer, it is worth reflecting on the relationship between brain and body. The brain is the organ of study for both psychiatry and neurology. This organ floats within the cerebrospinal fluid and is enclosed within the rigid, hard skull. However, the brain has myriad connections to the rest of the body, via the immune system, peripheral nervous system, gut-brain connection, etc. It has become increasingly clear that alterations in the regulation of systemic or body function can have powerful neuropsychiatric manifestations and alterations in brain function can have powerful effects on somatic function. This month's CNS Spectrums examines some of this cybernetic feedback between brain and body.

For example, little is known about psychiatric comorbidity in mitochondrial disease. In the first article, Omar Fattal, MD, and colleagues examine 36 subjects with mitochondrial cytopathies for the prevalence of psychiatric comorbidity by administering the Mini International Neuropsychiatric Interview and the Short Form-36 questionnaire. Surprisingly, lifetime psychiatric diagnoses for these patients included $54 \%$ major depressive disorder, $17 \%$ bipolar disorder, and $11 \%$ panic disorder. These prevalence rates were compared with the general population and subjects with cancer and epilepsy. Subjects with a comorbid psychiatric diagnosis were older, had more hospital admissions, more medical conditions, and lower quality of life than subjects with mitochondrial disease alone. Thus, clinicians caring for persons with mitochondrial cytopathies should note the high prevalence of psychiatric problems, and understand that this comorbidity might have etiological and therapeutic implications.

On the other hand, borderline personality disorder (BPD) is considered a brain-based disorder best left to psychiatrists, since these patients are among the most challenging of all patients to clinicians. One major hurdle to effective management of such patients is that they often do not follow their doctor's orders, and this contributes to the long-term disability of the disorder. Anything that might increase the compliance of such challenging patients would be a real boon to their long-term management.

Daphne Simeon, MD, and colleagues assess the efficacy and tolerability of a once-daily extended-release form of divalproex in 20 adult outpatients with Diagnostic and Statistical Manual of Mental Disorders, Fourth Edition, BPD via a 12-week open-label trial. Primary outcome measures included the Clinical Global Impression-Improvement scale and the Global Assessment Scale. Secondary outcome measures assessed aggression; affective disturbance; dissociation; and general psychopathology. Treatment was associated with statistically significant improvement on the Clinical Global Impression-Improvement scale, the Global Assessment Scale, the Overt Aggression ScaleModified irritability subscale, and the Aggression Questionaire. Seven out of 10 completers (70\%) were treatment responders. There was no significant decline in affective lability or in dissociation. Only one participant discontinued treatment due to adverse events. These findings support that divalproex extended-release is an efficacious and well-tolerated pharmacologic agent for $\mathrm{BPD}$, with the additional advantage of single daily dosing at bedtime. Placebo-controlled trials are needed for replication.

In addition to being a key target of personality disorders, impulsivity is also a major driving force in the impulse-control disorders, which have received relatively little attention from the mental health community. An increasing awareness of the prevalence and impact of these dis-

Dr. Hollander is the editor of this journal, Esther and Joseph Klingenstein Professor and Chairman of Psychiatry at the Mount Sinai School of Medicine, and director of the Seaver and New York Autism Center of Excellence in New York City. 
orders is emerging, yet still the impulse-control disorders are not included in national prevalence surveys, and funding agencies such as National Institute of Mental Health and National Institute on Drug Abuse have refused to include these common, disabling disorders as priority areas worthy of scant research funding.

Among impulse-control disorders, problematic Internet use has been considered and examined. Prevalence estimate studies indicate that problematic Internet use is experienced across geographic locations by many individuals of diverse backgrounds. Timothy Liu, MD, and Mark N. Potenza, MD, PhD, examine problematic Internet use from epidemiological and clinical perspectives. Clinicians should be familiar with the extent of problematic Internet use and the data regarding the efficacies and tolerabilities of currently available treatments.

On the other hand, compulsivity can be viewed as a symptom domain that cuts across various conditions, and may be expressed in a spectrum of related conditions. As such, it has been proposed that certain DSM-IV Axis I disorders share overlapping clinical features, genetic contributions and treatment response and fall within an "obsessive-compulsive" (OC) spectrum. Obsessive-compulsive personality disorder (OCPD) does seem to resemble obsessive-compulsive disorder (OCD) and other "spectrum" disorders in terms of phenomenology, comorbidity, neurocognition, and treatment response. Next, Naomi Fineberg, MBBS, MA, MRCPsych, and colleagues critically examine the nosological profile of OCPD with special reference to $O C D$ and related disorders. If we view OCPD as a candidate member of the OC spectrum, this will have important implications for understanding the neurobiology, etiology, and potential treatments of this relatively unexplored condition. This might also influence how OCPD is considered in the $D S M-V$ with the subsequent removal of the disorder from Axis II, and a repositioning of it as an Axis I OC-related disorder condition.

Finally, at the turn of the $19^{\text {th }}$ century, fever therapy (pyretotherapy) was often used as a treatment for psychosis. However, pyretotherapy fell out of use after the introduction of convulsive methods. Gabriele Sani, MD, and colleagues report on a case of schizoaffective disorder and review classical and recent literature on fever and psychosis. This patient developed auditory hallucinations, persecutory delusional ideas, and was terrified soon upon his arrival in a foreign country. After being treated for 12 days with olanzapine and haloperidol, he developed fever due to urinary infection; his creatine phosophokinase levels were high, prompting the authors to suspend the use of antipsychotics. Psychotic symptom resolution followed immediately after fever abatement. The time course of symptom resolution in this patient suggests that fever had a beneficial role in this case. The relationships between body temperature changes and psychotic symptoms need to be further studied.

I have personally observed that in some children with autism, the development of fever may be associated with a remarkable improvement in various autistic target symptoms. This too serves to remind me that somatic changes can sometimes be associated with marked alterations in the symptom expression of brain based disorders, further reinforcing the brain-body connection. CNS 